\title{
PHYSIOTHERAPIST STUDENTS' PERCEPTIONS IN A GAMIFICATION PROJECT
}

\author{
Carolina Jiménez-Sánchez ${ }^{1,2}$, Pablo Gargallo-Aguaron', María Pilar López-Royo ${ }^{1,2}$, \\ \& Fani Alfaro-Gervon ${ }^{1}$ \\ ${ }^{1}$ Department of Physiotherapy, Universidad San Jorge, Zaragoza (Spain) \\ ${ }^{2}$ Iphysio Research Group, Universidad San Jorge, Zaragoza (Spain)
}

\begin{abstract}
Motivation is a crucial aspect in the students' learning. Thus, active learning methods encourage students to become active protagonists of their own learning processes. Gamification is the use of game elements in a non-game context. The use of gamification as an innovative teaching-learning strategy rises from the need for change in teaching methodology. By applying gamification to the classroom, students could be motivated to learn in new ways or enjoy otherwise tedious tasks. For that reason, a gamification project entitled 'Jeopardy', based on a cooperative methodology, has been developed in the first semester of the academic course 2019-2020. The participants have been students of $2^{\circ}$ course of Physiotherapy degree from San Jorge University, enrolled in the subject 'General Procedures in Physiotherapy 1'. Jeopardy project consisted in the implementation of educational games that gave students, divided into small groups, tasks to earn points and feature a leaderboard to track progress and increase students' engagement. Our main objective was to analyze the perception of learners about their participation, the impact of the project and, the integration of content from this subject. In order to assess students' perceptions and satisfaction with the gamification project, an online survey was designed to collect quantitative and qualitative data. Results show that students value this project positively in terms of the way in which it facilitates the integration of content in a collaborative way. To conclude, this study provides that the use of a gamification project is a valid active learning strategy.
\end{abstract}

Keywords: Gamification, cooperative learning, active learning, physiotherapy.

\section{Introduction}

\subsection{Analysis of the initial situation}

Learning is the incorporation of new data and the ability to put it into practice when necessary and in a real environment (Lopea, 2000).

In addition, recent publications emphasize the need for creative work to acquire and apply new learning (Elisondo et al, 2011). In this model, teachers are decisive in designing, proposing and directing innovative projects that pose a creative and knowledge challenge for students. Therefore, the teacher becomes the coordinator, organizer, trainer and follower of the cooperative work of the students (Meroño \& Acuña, 2012).

Cooperative projects require a work team that must organize, participate and contribute to achieve a creative solution to a problem raised. Furthermore, Johnson and Johnson state that working through collaborative projects in small groups manages to develop interpersonal skills thanks to positive interdependence and the evaluation of group performance (Baker et al, 2018).

Nowadays, one strategy used in the classroom is gamification, defined as the use of the principles of the game in non-common contexts (Attali \& Arieli-Attali, 2015). It is a methodology where students can purchase new knowledge, reinforce them, experience simulations and/or solve real world problems in a playful way. In addition, it allows students to be introduced to collaborative environments, very close to real professional contexts where there are challenges. Furthermore, the fact that there is a return in the form of a qualification as a "reward or prize", favours the motivation of the students.

Although play and fun are not always associated with learning, many aspects of play and own games are very conductive to learning. Thus, educators have seen an increase in engagement and knowledge retention when using games (Bruder, 2015). 


\subsection{Innovation highlights and their justification}

Higher education is increasingly based on the development of competencies and interdisciplinarity facilitating both transversal skills and self-learning (Baños \& Perez, 2005). This model of higher education displaces the traditional figure of the teacher and student since the teachers, who are an interactive part of these new methodologies, Will have to develop new pedagogical strategies to achieve the implementation of both specific and transversal objectives and the student will have an active role in their learning (Alvarez, 2004).

The gamma activities increase interactivity and motivation. Moreover, much more so if there is a reward for their participation, involvement and collaboration (Sera \& Wheeler, 2017). Therefore, carrying out gamification activities in a collaborative manner will facilitate both the learning process and the development of skills inherent to the degree.

\section{Context of the intervention and objectives of the proposal}

The general objective of this project is to evaluate the impact of the implementation of a gamification project to favour the assimilation of the content of the General Procedures in Physiotherapy 1 (GPP-1) subject.

As specific objectives:

- To apply and extend specific knowledge of the content developed in the GPP-1 subject.

- To acquire and develop professional competences through the collaboration between the members of the groups.

- To work within a team participating in a collaborative way contributing knowledge and ideas for the resolution of the proposed gamification activities.

- To evaluate by means of appropriate tools, the influence of the Jeopardy project on the integration of the subject content.

- To evaluate, by means of appropriate tools, aspects related to the collaborative work during the development of the activity.

\section{Methods}

\subsection{Participants}

Students in the second year of the Physiotherapy degree of the Universidad San Jorge, enrolled in the 1 GPF-1 subject, participated in the project.

\subsection{Preparation and development of the gamification project}

At the beginning of the teaching period, lecturers instructed students on the project's operation and the scoring system. At the same time, students were divided into small groups of 14-16 students to encourage them to work as a team.

Jeopardy project was a set of evaluated tasks worth $10 \%$ of the final mark in GPP-1. These tasks included interactive games using different platforms, micro-classes, and activities based on problem solving as clinical cases. During each class the students worked on a specific topic carrying out the different activities. Each task was marked with a specific score.

The activity took place during the first semester of the academic course 2019-2020.

\subsection{Assessment}

In order to evaluate the student's perceptions about the learning process through Jeopardy project, the students were surveyed in the last class using the Intrinsic Motivation Inventory (IMI) scale.

The IMI includes 45 items measured on a 7-point Likert scale from 1 (strongly disagree) to 7 (strongly agree) and assesses issues such as participant's interest/enjoyment, perceived competence, effort/importance, pressure/tension, perceived choice, relatedness and value/usefulness while performing a given activity.

\section{Results and discussion}

122 students (53 males, 69 females) with a mean age of $20,97 \pm 3,56$ years participated in this project.

Table 1 below shows students' perceptions and considerations about their motivation of the Jeopardy project. 
Table 1. Intrinsic Motivation Inventory (IMI).

\begin{tabular}{|c|c|c|c|c|c|c|c|}
\hline SUBSCALE & \multicolumn{7}{|c|}{ ANSWER } \\
\hline Interest/Enjoyment & $\begin{array}{l}\text { Strongly } \\
\text { Disagree }\end{array}$ & Disagree & $\begin{array}{l}\text { Somewhat } \\
\text { Disagree }\end{array}$ & $\begin{array}{l}\text { Neither } \\
\text { Agree nor } \\
\text { Disagree } \\
\end{array}$ & $\begin{array}{l}\text { Somewhat } \\
\text { Agree }\end{array}$ & Agree & $\begin{array}{l}\text { Strongly } \\
\text { Agree }\end{array}$ \\
\hline $\begin{array}{l}\text { I enjoyed doing this } \\
\text { activity very much }\end{array}$ & $3,28 \%$ & $4,10 \%$ & $3,28 \%$ & $18,85 \%$ & $32,79 \%$ & $26,23 \%$ & $11,48 \%$ \\
\hline $\begin{array}{l}\text { This activity was fun to } \\
\text { do. }\end{array}$ & $1,64 \%$ & $4,92 \%$ & $6,56 \%$ & $9,02 \%$ & $27,05 \%$ & $36,89 \%$ & $13,93 \%$ \\
\hline $\begin{array}{l}\text { I thought this was a boring } \\
\text { activity. }(\mathrm{R})\end{array}$ & $4,10 \%$ & $13,93 \%$ & $16,39 \%$ & $17,21 \%$ & $16,39 \%$ & $24,59 \%$ & $7,38 \%$ \\
\hline $\begin{array}{l}\text { This activity did not hold } \\
\text { my attention at all. (R) }\end{array}$ & $4,92 \%$ & $9,84 \%$ & $10,66 \%$ & $17,21 \%$ & $12,30 \%$ & $27,87 \%$ & $17,21 \%$ \\
\hline $\begin{array}{l}\text { I would describe this } \\
\text { activity as very interesting. }\end{array}$ & $2,46 \%$ & $3,28 \%$ & $9,02 \%$ & $21,31 \%$ & $28,69 \%$ & $25,41 \%$ & $9,84 \%$ \\
\hline $\begin{array}{l}\text { I thought this activity was } \\
\text { quite enjoyable. }\end{array}$ & $1,64 \%$ & $5,74 \%$ & $7,38 \%$ & $25,41 \%$ & $27,87 \%$ & $23,77 \%$ & $8,20 \%$ \\
\hline $\begin{array}{l}\text { While I was doing this } \\
\text { activity, I was thinking } \\
\text { about how much I enjoyed } \\
\text { it. }\end{array}$ & $7,38 \%$ & $5,74 \%$ & $11,48 \%$ & $20,49 \%$ & $29,51 \%$ & $18,85 \%$ & $6,56 \%$ \\
\hline Perceived Competence & $\begin{array}{l}\text { Strongly } \\
\text { Disagree }\end{array}$ & Disagree & $\begin{array}{l}\text { Somewhat } \\
\text { Disagree }\end{array}$ & $\begin{array}{l}\text { Neither } \\
\text { Agree Nor } \\
\text { Disagree }\end{array}$ & $\begin{array}{l}\text { Somewhat } \\
\text { Agree }\end{array}$ & Agree & $\begin{array}{l}\text { Strongly } \\
\text { Agree }\end{array}$ \\
\hline $\begin{array}{l}\text { I think I am pretty good at } \\
\text { this activity. }\end{array}$ & $0,82 \%$ & $6,56 \%$ & $18,03 \%$ & $36,07 \%$ & $18,03 \%$ & $14,75 \%$ & $5,74 \%$ \\
\hline $\begin{array}{l}\text { I think I did pretty well at } \\
\text { this activity, compared to } \\
\text { other students. }\end{array}$ & $0,00 \%$ & $13,93 \%$ & $16,39 \%$ & $29,51 \%$ & $22,95 \%$ & $11,48 \%$ & $5,74 \%$ \\
\hline $\begin{array}{l}\text { After working at this } \\
\text { activity for a while, I felt } \\
\text { pretty competent. }\end{array}$ & $0,82 \%$ & $9,02 \%$ & $20,49 \%$ & $30,33 \%$ & $26,23 \%$ & $7,38 \%$ & $5,74 \%$ \\
\hline $\begin{array}{l}\text { I am satisfied with my } \\
\text { performance at this task. }\end{array}$ & $0,82 \%$ & $8,20 \%$ & $13,93 \%$ & $26,23 \%$ & $22,95 \%$ & $17,21 \%$ & $10,66 \%$ \\
\hline $\begin{array}{l}\text { I was pretty skilled at this } \\
\text { activity. }\end{array}$ & $1,64 \%$ & $9,84 \%$ & $15,57 \%$ & $37,70 \%$ & $20,49 \%$ & $7,38 \%$ & $7,38 \%$ \\
\hline Effort/Importance & $\begin{array}{l}\text { Strongly } \\
\text { Disagree }\end{array}$ & Disagree & $\begin{array}{l}\text { Somewhat } \\
\text { Disagree }\end{array}$ & $\begin{array}{l}\text { Neither } \\
\text { Agree Nor } \\
\text { Disagree }\end{array}$ & $\begin{array}{l}\text { Somewhat } \\
\text { Agree }\end{array}$ & Agree & $\begin{array}{l}\text { Strongly } \\
\text { Agree }\end{array}$ \\
\hline $\begin{array}{l}\text { I put a lot of effort into } \\
\text { this. }\end{array}$ & $1,64 \%$ & $9,84 \%$ & $17,21 \%$ & $27,87 \%$ & $18,03 \%$ & $18,03 \%$ & $7,38 \%$ \\
\hline $\begin{array}{l}\text { I did not try very hard to } \\
\text { do well at this activity. (R) }\end{array}$ & $3,28 \%$ & $6,56 \%$ & $16,39 \%$ & $31,15 \%$ & $13,11 \%$ & $20,49 \%$ & $9,02 \%$ \\
\hline $\begin{array}{l}\text { I tried very hard on this } \\
\text { activity. }\end{array}$ & $0,00 \%$ & $10,66 \%$ & $17,21 \%$ & $27,87 \%$ & $20,49 \%$ & $16,39 \%$ & $7,38 \%$ \\
\hline $\begin{array}{l}\text { It was important to me to } \\
\text { do well at this task. }\end{array}$ & $0,00 \%$ & $4,10 \%$ & $2,46 \%$ & $13,93 \%$ & $27,87 \%$ & $31,97 \%$ & $19,67 \%$ \\
\hline $\begin{array}{l}\text { I did not put much energy } \\
\text { into this. }(\mathrm{R})\end{array}$ & $4,10 \%$ & $8,20 \%$ & $13,11 \%$ & $30,33 \%$ & $13,11 \%$ & $18,03 \%$ & $13,11 \%$ \\
\hline Pressure/Tension & $\begin{array}{l}\text { Strongly } \\
\text { Disagree }\end{array}$ & Disagree & $\begin{array}{l}\text { Somewhat } \\
\text { Disagree }\end{array}$ & $\begin{array}{l}\text { Neither } \\
\text { Agree nor } \\
\text { Disagree } \\
\end{array}$ & $\begin{array}{l}\text { Somewhat } \\
\text { Agree }\end{array}$ & Agree & $\begin{array}{l}\text { Strongly } \\
\text { Agree }\end{array}$ \\
\hline $\begin{array}{l}\text { I did not feel nervous at all } \\
\text { while doing this. (R) }\end{array}$ & $17,21 \%$ & $26,23 \%$ & $13,93 \%$ & $18,03 \%$ & $13,93 \%$ & $6,56 \%$ & $4,10 \%$ \\
\hline $\begin{array}{l}\text { I felt very tense while } \\
\text { doing this activity. }\end{array}$ & $16,39 \%$ & $27,87 \%$ & $11,48 \%$ & $24,59 \%$ & $10,66 \%$ & $4,10 \%$ & $4,92 \%$ \\
\hline $\begin{array}{l}\text { I was very relaxed in } \\
\text { doing these. }(\mathrm{R})\end{array}$ & $14,75 \%$ & $20,49 \%$ & $22,13 \%$ & $17,21 \%$ & $14,75 \%$ & $8,20 \%$ & $2,46 \%$ \\
\hline $\begin{array}{l}\text { I was anxious while } \\
\text { working on this task. }\end{array}$ & $18,03 \%$ & $23,77 \%$ & $9,02 \%$ & $30,33 \%$ & $10,66 \%$ & $4,10 \%$ & $4,10 \%$ \\
\hline $\begin{array}{l}\text { I felt pressured while } \\
\text { doing these }\end{array}$ & $18,85 \%$ & $26,23 \%$ & $12,30 \%$ & $26,23 \%$ & $9,02 \%$ & $2,46 \%$ & $4,92 \%$ \\
\hline
\end{tabular}




\begin{tabular}{|c|c|c|c|c|c|c|c|}
\hline Perceived Choice & $\begin{array}{l}\text { Strongly } \\
\text { Disagree }\end{array}$ & Disagree & $\begin{array}{l}\text { Somewhat } \\
\text { Disagree }\end{array}$ & $\begin{array}{l}\text { Neither } \\
\text { Agree nor } \\
\text { Disagree }\end{array}$ & $\begin{array}{l}\text { Somewhat } \\
\text { Agree }\end{array}$ & Agree & $\begin{array}{l}\text { Strongly } \\
\text { Agree }\end{array}$ \\
\hline $\begin{array}{l}\text { I believe I had some } \\
\text { choice about doing this } \\
\text { activity. }\end{array}$ & $11,48 \%$ & $12,30 \%$ & $6,56 \%$ & $31,97 \%$ & $11,48 \%$ & $18,85 \%$ & $7,38 \%$ \\
\hline $\begin{array}{l}\text { I felt like it was not my } \\
\text { own choice to do this } \\
\text { task. (R) }\end{array}$ & $15,57 \%$ & $15,57 \%$ & $13,93 \%$ & $25,41 \%$ & $7,38 \%$ & $14,75 \%$ & $7,38 \%$ \\
\hline $\begin{array}{l}\text { I did not really have a } \\
\text { choice about doing this } \\
\text { task. (R) }\end{array}$ & $16,39 \%$ & $13,93 \%$ & $9,02 \%$ & $31,15 \%$ & $6,56 \%$ & $13,93 \%$ & $9,02 \%$ \\
\hline $\begin{array}{l}\text { I felt like I had to do this. } \\
\text { (R) }\end{array}$ & $15,57 \%$ & $25,41 \%$ & $23,77 \%$ & $26,23 \%$ & $4,92 \%$ & $2,46 \%$ & $1,64 \%$ \\
\hline $\begin{array}{l}\text { I did this activity because } \\
\text { I had no choice. (R) }\end{array}$ & $13,93 \%$ & $22,95 \%$ & $21,31 \%$ & $22,13 \%$ & $4,10 \%$ & $9,84 \%$ & $5,74 \%$ \\
\hline $\begin{array}{l}\text { I did this activity because } \\
\text { I wanted to. }\end{array}$ & $6,56 \%$ & $4,92 \%$ & $11,48 \%$ & $29,51 \%$ & $18,85 \%$ & $22,13 \%$ & $6,56 \%$ \\
\hline $\begin{array}{l}\text { I did this activity because } \\
\text { I had to. }(\mathrm{R})\end{array}$ & $23,77 \%$ & $22,95 \%$ & $25,41 \%$ & $18,85 \%$ & $2,46 \%$ & $3,28 \%$ & $3,28 \%$ \\
\hline Relatedness & $\begin{array}{l}\text { Strongly } \\
\text { Disagree }\end{array}$ & Disagree & $\begin{array}{l}\text { Somewhat } \\
\text { Disagree }\end{array}$ & $\begin{array}{l}\text { Neither } \\
\text { Agree nor } \\
\text { Disagree }\end{array}$ & $\begin{array}{l}\text { Somewhat } \\
\text { Agree }\end{array}$ & Agree & $\begin{array}{l}\text { Strongly } \\
\text { Agree }\end{array}$ \\
\hline $\begin{array}{l}\text { I felt really distant to this } \\
\text { person. }(\mathrm{R})\end{array}$ & $6,56 \%$ & $5,74 \%$ & $13,11 \%$ & $16,39 \%$ & $6,56 \%$ & $27,05 \%$ & $24,59 \%$ \\
\hline $\begin{array}{l}\text { I really doubt that this } \\
\text { person and I would ever } \\
\text { be friends. (R) }\end{array}$ & $6,56 \%$ & $5,74 \%$ & $13,11 \%$ & $22,95 \%$ & $7,38 \%$ & $23,77 \%$ & $20,49 \%$ \\
\hline $\begin{array}{l}\text { I felt like I could really } \\
\text { trust this person. }\end{array}$ & $3,28 \%$ & $8,20 \%$ & $10,66 \%$ & $27,05 \%$ & $22,13 \%$ & $13,93 \%$ & $14,75 \%$ \\
\hline $\begin{array}{l}\text { I'd like a chance to } \\
\text { interact with this person } \\
\text { more often. }\end{array}$ & $1,64 \%$ & $4,10 \%$ & $3,28 \%$ & $29,51 \%$ & $27,87 \%$ & $22,13 \%$ & $11,48 \%$ \\
\hline $\begin{array}{l}\text { I'd really prefer not to } \\
\text { interact with this person } \\
\text { in the future. }(\mathrm{R})\end{array}$ & $3,28 \%$ & $4,10 \%$ & $4,10 \%$ & $24,59 \%$ & $5,74 \%$ & $21,31 \%$ & $36,89 \%$ \\
\hline $\begin{array}{l}\text { I don't feel like I could } \\
\text { really trust this person. } \\
\text { (R) }\end{array}$ & $3,28 \%$ & $3,28 \%$ & $9,84 \%$ & $22,13 \%$ & $10,66 \%$ & $24,59 \%$ & $26,23 \%$ \\
\hline $\begin{array}{l}\text { It is likely that this person } \\
\text { and I could become } \\
\text { friends if we interacted a } \\
\text { lot. }\end{array}$ & $0,82 \%$ & $3,28 \%$ & $7,38 \%$ & $23,77 \%$ & $22,95 \%$ & $22,13 \%$ & $19,67 \%$ \\
\hline I feel close to this person. & $3,28 \%$ & $9,84 \%$ & $9,02 \%$ & $33,61 \%$ & $23,77 \%$ & $10,66 \%$ & $9,84 \%$ \\
\hline Value/Usefulness & $\begin{array}{l}\text { Strongly } \\
\text { Disagree }\end{array}$ & Disagree & $\begin{array}{l}\text { Somewhat } \\
\text { Disagree }\end{array}$ & $\begin{array}{l}\text { Neither } \\
\text { Agree nor } \\
\text { Disagree } \\
\end{array}$ & $\begin{array}{l}\text { Somewhat } \\
\text { Agree }\end{array}$ & Agree & $\begin{array}{l}\text { Strongly } \\
\text { Agree }\end{array}$ \\
\hline $\begin{array}{l}\text { I believe this activity } \\
\text { could be of some value to } \\
\text { me. }\end{array}$ & $3,28 \%$ & $5,74 \%$ & $5,74 \%$ & $18,03 \%$ & $25,41 \%$ & $31,97 \%$ & $9,84 \%$ \\
\hline $\begin{array}{l}\text { I would be willing to do } \\
\text { this again because it has } \\
\text { some value to me. }\end{array}$ & $5,74 \%$ & $5,74 \%$ & $9,02 \%$ & $19,67 \%$ & $27,05 \%$ & $22,13 \%$ & $10,66 \%$ \\
\hline $\begin{array}{l}\text { I think this is an } \\
\text { important activity. }\end{array}$ & $2,46 \%$ & $7,38 \%$ & $5,74 \%$ & $17,21 \%$ & $27,05 \%$ & $28,69 \%$ & $11,48 \%$ \\
\hline
\end{tabular}

All these findings align with literature on the difficulty of finding student motivation and engagement (Hanus \& Fox, 2015). Despite this, the entertainment value of the games in education makes they more enjoyable than other learning activities. Moreover, the contemporary students need interest, engagement, stimulation, and entertainment to support their learning (Day-Black et al., 2015).

Regarding the perceived competence subscale, it is theorized to be a positive predictor of behavioural measure of intrinsic motivation. In view of our results, there was generally a high level of satisfaction about their individual performance. However, Zichermann (2011) argued that intrinsic motivation is unreliable, and it is necessary using extrinsic motivation as incentives as we have applied in our study. 
Some of the advantages of this type of gamified activity is that learners must review contents prior to each activity and therefore, this involves considerable effort and dedication (Hanus \& Fox, 2015), as reflected in the effort/importance subscale.

Pressure/Tension is theorized to be a negative predictor of intrinsic motivation and evaluates if participants feel pressure to succeed in an activity (Monteiro, Mata \& Peixoto, 2015). This subscale was not scored with higher scores, so there was no evidence of an important pressure.

Other factor such as perceived choice was assessed. In this regard, it should be noted that despite the project was an obligatory activity, it was perceived neither as a forced activity. On the other hand, another aspect that is interesting to highlight is the complex interaction of students within-groups since groups were not organized by own students (relatedness subscale).

About the usefulness of the gamification, this project was positively valued by a large majority of the students. This approach must be integrated in a way that reinforces students' perception of the importance of education for the professional future (Urh et al., 2015).

To conclude, findings from this study revealed that the application of a gamification project has been recognised as an interesting learning methodology although students found some disadvantages such as an important effort and some difficulties in relationship to their peers.

\section{References}

Alvarez, R.P. (2004). Formación superior basada en competencias, interdisciplinariedad y trabajo autónomo del estudiante. Revista iberoamericana de educación, 35.

Attali, Y., \& Arieli-Attali, M. (2015). Gamification in assessment: Do points affect test performance? Computers \& Education, 83, 57-63.

Baker. B., Drane, C., Chambers, J., \& Lindqvist, S. (2018). Training students as interprofessional learning facilitators: an exploratory study highlighting the need to build confidence. Journal of interprofessional care, 32(4), 473-480.

Baños, J., \& Pérez, J. (2005). Cómo fomentar las competencias transversales en los estudios de Ciencias de la Salud: una propuesta de actividades. Educación Médica, 8(4), 40-49.

Bruder, P. (2015). Game on: Gamification in the classroom. The Education Digest, 3, 56-60.

Day-Black, C., Merrill, E.B., Konzelman, L., Williams, T.T., \& Hart, N. (2015). Gamification: An Innovative Teaching-Learning Strategy for the Digital Nursing Students in a Community Health Nursing Course. ABNF J, 26(4), 90-4.

Elisondo, R., Rinaudo, M.C., \& Donolo, D. (2011). Actividades inesperadas como oportunidades para la creatividad. Innovación Educativa, 11(57), 147-155.

Hanus, M.D., \& Fox, J. (2015). Assessing the effects of gamification in the classroom: A longitudinal study on intrinsic motivation, social comparison, satisfaction, effort, and academic performance. Computers \& Education, 80, 152-161.

Lopea, L.F. (2000). De la teoría general a la enseñanza en el aula. Sinéctica, 17, 39-51.

Meroño, M.C., Acuña, B.P. (2012). El aprendizaje cooperativo mediante actividades participativas. Anales de la Universidad Metropolitana, 12(2).

Monteiro, V., Mata, L., \& Peixoto, F. (2015). Intrinsic Motivation Inventory: Psychometric Properties in the Context of First Language and Mathematics Learning. Psychology/Psicologia Reflexão e Crítica, 28(3), 434-443.

Sera, L., \& Wheeler, E. (2017). Game on: The gamification of the pharmacy classroom. Currents in Pharmacy Teaching and Learning, 9, 155-159.

Urh, M., Vukovic G., Jereb, E., \& Pintar R. (2015). The model for introduction of gamification into e-learning in higher education. Procedia - Social and Behavioral Sciences, 197, 388-397.

Zichermann, G., (2011). Intrinsic and Extrinsic Motivation in Gamification. Retrieved from https://www.gamification.co/2011/10/27/intrinsic-and-extrinsic-motivation-in-gamification/ 